\title{
CO3D, A WORLDWIDE ONE-METER ACCURACY DEM FOR 2025
}

\author{
L. Lebègue, E. Cazala-Hourcade, F. Languille, S. Artigues, O. Melet \\ CNES, 18 avenue Edouard Belin, 31401 Toulouse, France - (laurent.lebegue, eric.cazala-hourcade, florie.languille, \\ stephanie.artigues, olivier.melet)@cnes.fr
}

Commission I, WG I/8

KEY WORDS: DEM, constellation, image quality, satellites, photogrammetry

\begin{abstract}
:
The goal of the CO3D (Constellation Optique 3D) mission is the full-automatic production of a worldwide accurate DEM. CO3D is also a constellation of a new generation of low-cost optical satellites. The DEM accuracy is expected to be one meter in relative height and two meters in absolute height with a one-meter grid space. Each of the four satellites of the constellation will provide images with $0.50 \mathrm{~m}$ resolution in red, green, blue bands. A NIR (Near-InfraRed) band will also be available with a resolution close to $1 \mathrm{~m}$. The satellites resource will be shared by, on one hand, the French institutions (government, scientists concerned by global Earth monitoring) who will have dedicated access and preferred price conditions, and on the other hand commercial customers interested in 2D and 3D products. The launch of the constellation is expected mid-2023 and $90 \%$ of the DEM worldwide production should be reached by the end of 2025 .

Starting from an overview of the system characteristics and its main innovations, this paper presents the expected performance, the 2D and 3D products that should be available for the end-users and finally how they should be qualified.
\end{abstract}

\section{INTRODUCTION}

The CO3D mission, imagined by the French Space Agency (CNES), aims at providing a Digital Surface Model (DSM) of the world landmass, between $\mathrm{S} 60^{\circ}$ and $\mathrm{N} 70^{\circ}$, at low cost with a high accuracy of one meter in relative and two meters in absolute altimetry. The low-cost shall be reached thanks to fullautomatic production based on massive cloud processing. The accuracy shall be reached thanks to a constellation of a new generation of low-cost optical satellites providing $50 \mathrm{~cm}$ Ground Sampling Distance (GSD) colour imagery. The satellites will be built and operated by Airbus Defence and Space (ADS).

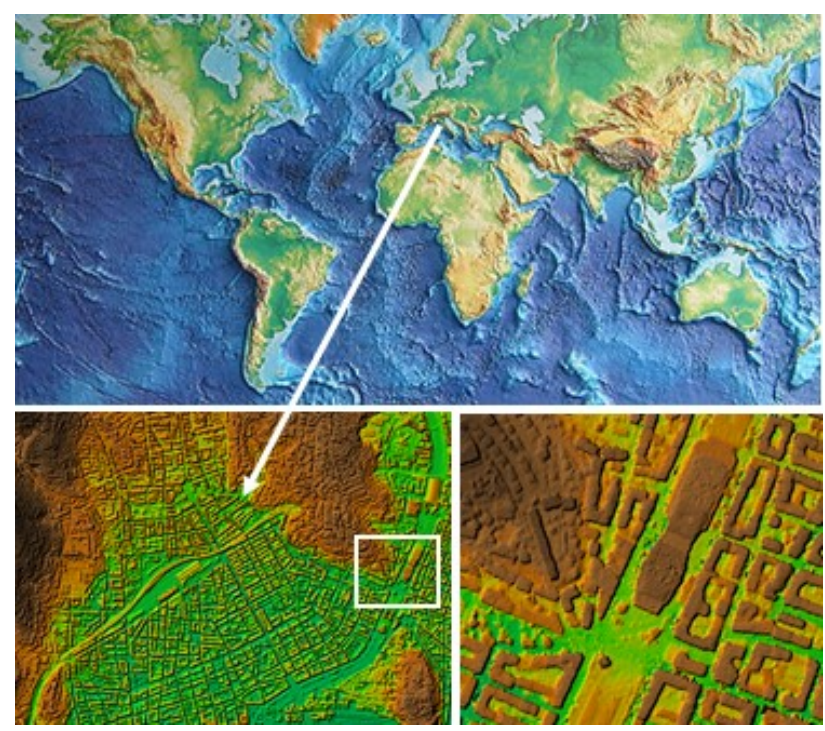

Figure 1. A one-meter accuracy worldwide DEM of landmasses between $\mathrm{S} 60^{\circ}$ and $\mathrm{N} 70^{\circ}$
The satellites resource will be shared by, on one hand, the French institutions (government, scientists), defining the core mission, who will have dedicated access and preferred price conditions, and on the other hand, ADS GEO, the future commercial operator, and its customers interested in 2D and 3D products. Many scientists concerned by global Earth monitoring have been interviewed about their needs in several studies to converge towards the system characteristics and the final products. For instance, glaciologists are interested in glaciers melting dynamic, snow scientists in mountains snow height and equivalent water volume assessment, geologists in landslide and reefs movements, French institute of cartography (IGN) in maps update.

The so-called 'PACA benchmark' survey gathered such users to train on CO3D likewise 3D datasets. Pleiades stereo acquisitions covering about $10000 \mathrm{~km}^{2}$ in the south of France have been processed on the cloud with a CNES 3D pipeline prototype based upon CARS (Youssefi 2020, Michel 2020) and, in parallel, with the IGN MicMac pipeline, to generate a DSM over the area. CNES, with the support of IGN, assessed the quality of the outputs and how they suit to the products user needs in terms of accuracy and formats. Most of the algorithms of the CNES 3D demonstrator pipeline will be included in the future $\mathrm{CO} 3 \mathrm{D} 3 \mathrm{D}$ production pipeline.

The CO3D mission lifetime, expected at least 5 years, will be split in two main phases. The first one, called E2p, is a one-year demonstration period dedicated to the assessment of the system performances over two areas: France and a $27 \mathrm{Mkm}^{2}$ governmental Arc of Interest covering the north of Africa and Middle-East countries.

The main performances concerned are the DSM quality (absolute altimetry will be checked over France thanks to IGN benchmarks), the stereo image collecting capacity of the constellation, and the $3 \mathrm{D}$ products processing time on the cloud. During this period, the worldwide acquisitions and productions will have started as well as the commercial ones with a lower priority. 


\section{Metropolitan FRANCE $500000 \mathrm{~km} 2$}

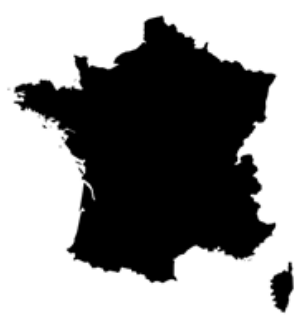

Arc Of Interest $27 \mathrm{Mkm} 2$

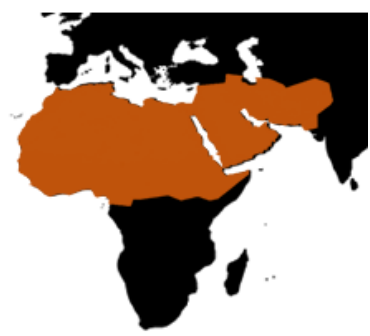

Figure 2. E2p demonstration areas

The second phase, called E2c, follows the E2p phase and ends with the mission lifetime. This period is dedicated to commercial activities with still resource sharing with the French institutions needs respecting accorded quota and priorities.

Concerning the global mission schedule, the launch of the constellation is forecast mid-2023, followed by a 3 months InOrbit Test (IOT) period (including Image Quality commissioning) and then, the E2p and E2c phases. As $90 \%$ of the world acquisitions should be realized within the two-years following the IOT, we can forecast the availability of a large part of the worldwide DEM by the end of 2025. At this paper redaction date, the project is close to end a nine-month CNESADS co-engineering definition phase, and most of the specification are established.

\section{SYSTEM CHARACTERISTICS}

\subsection{Spatial segment}

The CO3D spatial segment is based on a constellation of at least 4 VHR optical satellites, designed and built by ADS, "flying" on the same sun-synchronous orbital plane and working by pair to provide simultaneous stereo images. The orbit altitude is 502 $\mathrm{km}$ and the local mean time should be close to eleven o'clock to limit the size of spread shadows and cloud-cover. Four satellites can be launched in the same VEGA-C rocket shared with another partner.

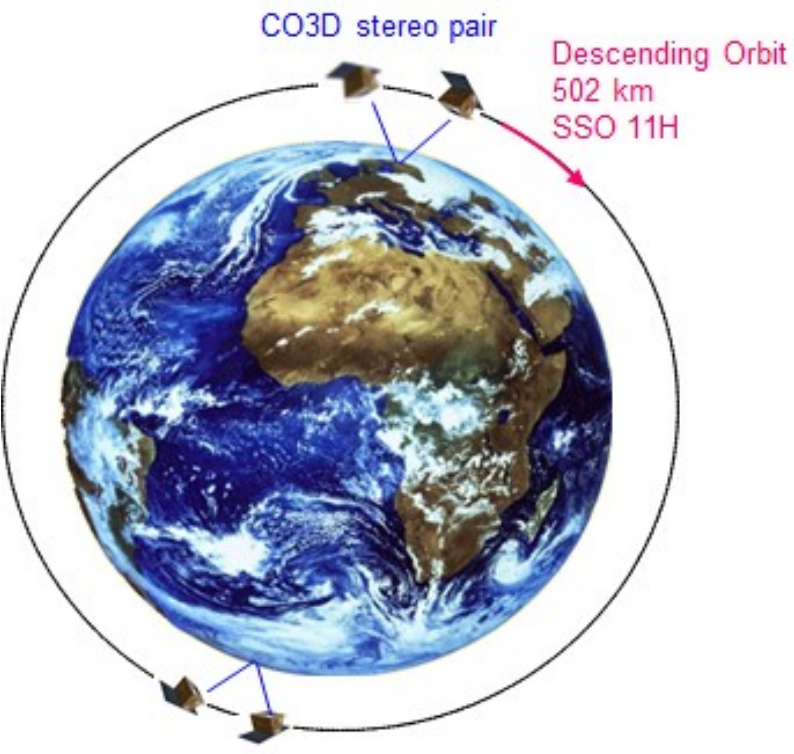

Figure 3. CO3D orbital configuration for 4 satellites
Images will be acquired in RGB spectral bands with a $50 \mathrm{~cm}$ GSD and in an additional Near-Infrared (Nir) band, with a GSD comprised between 0.50 and $1 \mathrm{~m}$, thanks to COTS matrix sensors. The footprint of each sensor is $7 \mathrm{~km} \times 5 \mathrm{~km}$ and the satellite agility allows $21 \times 25 \mathrm{~km}^{2}$ dynamic acquisition patterns.
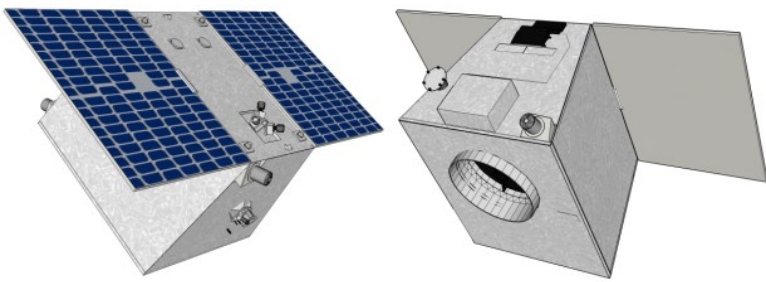

Figure 4. CO3D satellite views

\subsection{Ground segment}

CO3D satellites and their downlinked data will be operated from a Ground Segment that will include a Control Ground Segment (CGS) operated by ADS Space Systems and a User's Service Segment (USS) exploited by ADS GEO and its partners. The CO3D Ground Segment architecture is integrated within the multi-mission Geo-Digital Systems (GDS) of ADS GEO which already manages the ADS EO Constellation: SPOT6/Azersky, Pléiades and Pléiades Neo satellites.

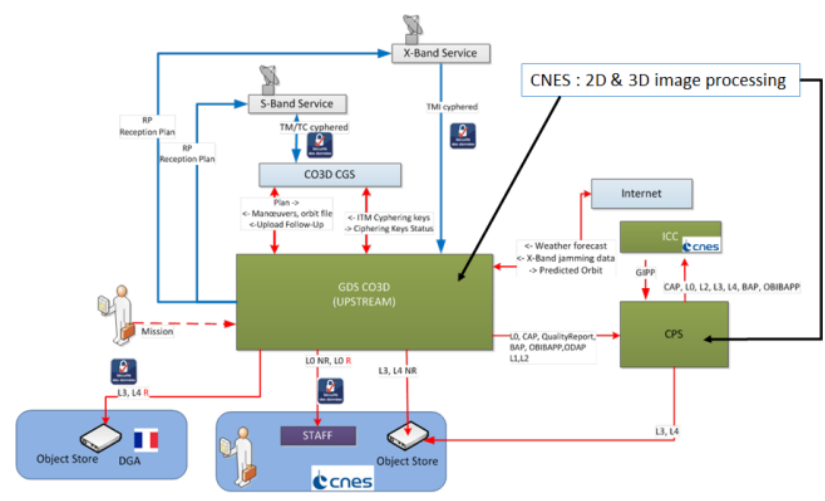

Figure 5. CO3D Ground Segment Overview

CNES is in charge of developing and delivering the 2D and 3D productions pipeline, as described by Melet (2020), that will be integrated by ADS GEO in its Ground Segment, but is also in charge of the development and exploitation during the E2p phase, of the Image Calibration Centre (ICC) that aggregate facilities to conduct the Image Quality commissioning.

\subsection{Main innovations}

The CO3D program gathers many innovations. The mission in itself is innovative because a worldwide DEM with such an accuracy at low-cost doesn't yet exist. The full-automatic processing of such a product is a real challenge and is conceivable thanks to the CNES long experience in 3D algorithms and massive cloud-computing.

Another innovation concerns the satellite constellation scheme that offers new flexibility in geometrical acquisition configurations to enhance the DSM generation. For instance, as most of moderate slope terrain will be acquired in stereo mode 
with a $0.30 \mathrm{~B} / \mathrm{H}$, high relief could be acquired with a $0.20 \mathrm{~B} / \mathrm{H}$ and main cities could be acquired in quadri-stereo respecting the so-called 'Diamond Geometry'. This geometry impose rather low $\mathrm{B} / \mathrm{H}$ suited to this kind of scenery and a variety of azimuthal viewing angles between the four views to reduce the occlusions coming from building and high relief.

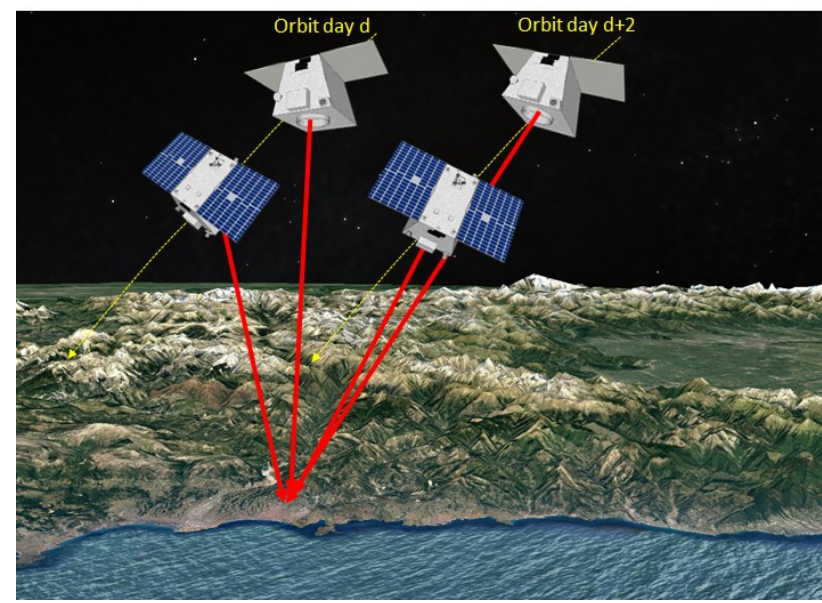

Figure 6. CO3D 'Diamond' geometry acquisitions

Another benefit of stereo imaging coming from two separate satellites is the opportunity to synchronize the two acquisitions with different viewing angles. The CO3D design has taken into account this capacity to reach a synchronization better than 30 ms between the two satellites of a stereo pair for a same target on the ground. Hence, moving objects, typically slower than 50 $\mathrm{km} / \mathrm{h}$, will be seen at the same position in the two images and can be reconstructed in the $3 \mathrm{D}$ processing. Moreover, these objects will not create ambiguous or false high objects in the $3 \mathrm{D}$ models when moving along the epipolar direction. This opportunity could also reveal new applications such as the $3 \mathrm{D}$ reconstruction of water surface or clouds volume.

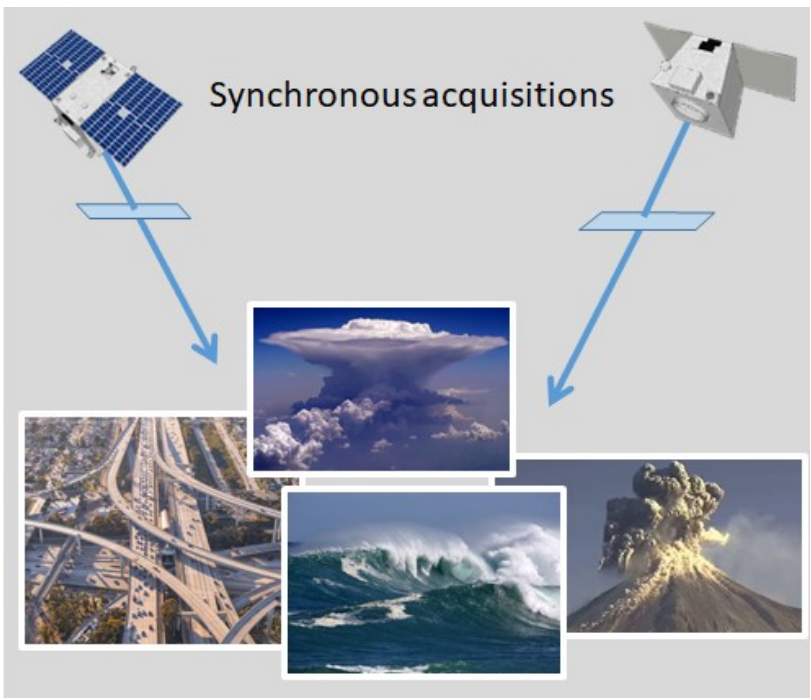

Figure 7. Acquisition synchronicity for better $3 \mathrm{D}$ reconstruction

The low-cost satellites are also full of innovations as they mix COTS matrix sensors and new AOCS device to allow efficient high-resolution images collecting with great agility.

Others innovations concern the end-user products described later, such as the dated DSM and 2D and 3D coherent series datasets enabling brand new change detection and mosaicing applications.

\section{SYSTEM PERFORMANCES}

\subsection{Altimetric accuracy}

The altimetric performance is a key factor of the CO3D mission. The relative altimetric requirement is given better than $1 \mathrm{~m}$ LE90 for slopes smaller than $5 \%$ and $1.5 \mathrm{~m}$ for higher slopes for $1 \mathrm{~m}$ grid spacing DSM. The main contributors to this performance are the accuracies of the correlation process and of the line-of-sight attitude restoration embedded in the geometric model.

The correlation algorithm used in the CARS software is PANDORA, as described by Cournet (2020). It is based on SGM, as designed by Hirschmuller (2008), and proved is efficiency in the PACA benchmark. On difficult terrain, such as urban areas, multiple DSM will be merged thanks to quadristereo acquisitions in order to reduce occlusions and noise in the $3 \mathrm{D}$ restoration process.

Concerning the satellite attitude, the worst situation would be residual micro-vibrations or drifts that would create waves on the final DSM as we can find on some existing products. Many means are performed to avoid these residuals. First, on board, the platform structure is designed to reduce the micro-vibration of the line-of-sight in magnitude and also in frequencies so that the high frequency gyroscope can measure most of its component. Then, several ground processings are implemented to assess potential residuals not restored by the gyroscope and update the geometric model with the corrections.

The absolute altimetric requirement is given better than $2 \mathrm{~m}$ LE90 for slopes smaller than $5 \%$ and $4 \mathrm{~m}$ for higher slopes for $1 \mathrm{~m}$ grid spacing DSM. This performance should be reached thanks to the refining of the geometric model on the Space Reference Point (SRP) database, described by Chandelier (2020), and also thanks to bundle-adjustment applied on large set of images. The SRP database GCP accuracy is given better than $3 \mathrm{~m}$ CE90 for planimetry and altimetry. Hence, the planimetric accuracy of the CO3D products is expected better than $5 \mathrm{~m}$ CE90 when enough GCP are available for the considered scenes. Otherwise the refining will be performed on the ADS GEO Premium Accuracy Services (PAS) orthoreference database whose planimetric accuracy is given better than $8 \mathrm{~m} \mathrm{CE90.} \mathrm{As} \mathrm{the} \mathrm{absolute} \mathrm{altimetric} \mathrm{accuracy} \mathrm{is} \mathrm{directly}$ related to the planimetric accuracy depending on the viewing angles, the CO3D acquisition plan limits roll angles to $15^{\circ}$ and pitch angles to $20^{\circ}$ for each satellite of a stereo pair. The last point, currently under discussion, would be to secure the performance by refining the DSM absolute altimetry thanks to very accurate IceSat- 2 data in the final process.

\subsection{Coverage}

The other key factor of the mission is the coverage capacity of the constellation. The main requirements concern the CO3D core mission. During the E2p phase, $90 \%$ of metropolitan France territory and $70 \%$ of the world landmass has to be completed. Concerning the Arc of Interest, which contains many difficult weather areas, $80 \%$ has to be completed in less than 18 months. Many simulations have been run with the CNES and ADS long-term mission simulators. These tools take into account cloud-coverage observation data from Meteofrance, the orbital configuration of the constellation, the satellite acquisition capacity such as agility, swath, memory 
storage volume, telemetry download rate, and also ground station network characteristics.

Other sets of simulations include an order book gathering institutional and commercial $2 \mathrm{D}$ and $3 \mathrm{D}$ user requests. For instance, the acquisition of a hundreds of glaciers during summer and winter, landslides sites and volcanos four times a year, the main cities in quadri-stereo, the largest harbour every week or the 2000 main airports twice a year. These simulations are very useful to optimize the acquisition strategy to fulfil most of the requests, tuning some parameters such as the priority levels, the strips length, or the area clustering.

The current simulations show, for the core mission, the Arc of Interest coverage completion given by Figure 8. On this Figure we can see that $75 \%$ of coverage is performed within one year and $90 \%$ in 18 months as required by the mission specification.

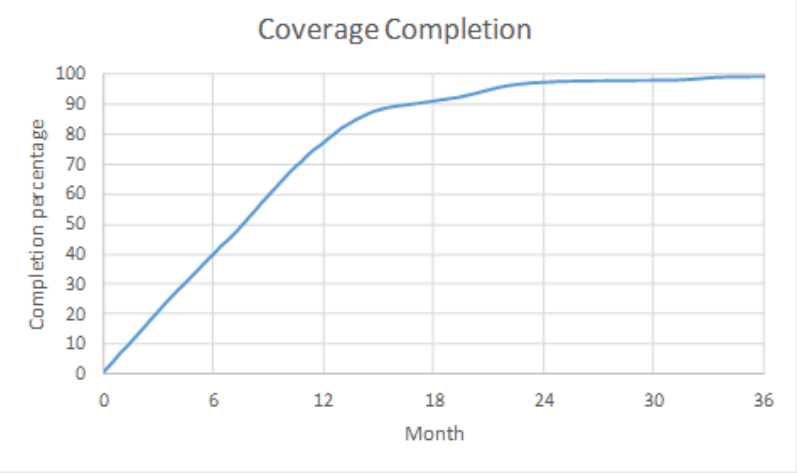

Figure 8. Arc of Interest coverage completion

\subsection{Processing time.}

To achieve the objectives of the CO3D mission in term of DSM production, a new image processing pipeline prototype is being developed by CNES (Melet et al, 2020) and used as part of the so-called "PACA benchmark". This new image processing pipeline takes advantage of big data technologies and is benchmarked with DSM production at a large scale with the socalled "PACA set".

The "PACA set" is set of 25 stereo and tri-stereo images from the High Resolution Pléiades satellites covering a large area of south-eastern France (PACA region). The "PACA set" cover about $10000 \mathrm{~km}^{2}$. The covered area has the advantage of containing a wide variety of reliefs: coastline edges, plain, urban areas (even very urban with Monaco and Nice) and mountains (Alps). This diversity is very interesting to test the performances of the processing pipeline on different situations. Mass production tests are carried out to validate the ability of the prototype to process DSM at a large scale.

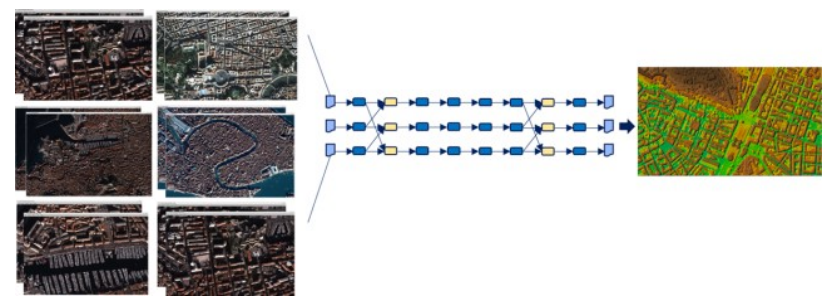

Figure 9. Paradigm for parallelization

The results obtained are as good as expected from an image quality point of view. The parallelization paradigm implemented using Big Data technologies makes the processing pipeline enough scalable for a mass production. This processing pipeline prototype will be the first release of the DSM production chain of the $\mathrm{CO} 3 \mathrm{D}$ program.

In the PACA benchmark, the Cloud cluster used in mass production test is composed of 26 computer nodes with 16vCPU and 60GB each. Each Spark job downloads needed data for computation from a cloud object storage and uploads the result in the same object storage. The total computation time to generate the DSM tiles of the entire PACA set is about 14 hours on the Cloud cluster. The processing time is consistent with the specification of the CO3D mission.

\section{END-USER PRODUCTS}

The CO3D system will provide end-users with $2 \mathrm{D}$ and $3 \mathrm{D}$ products to fulfil the core mission requests.

\subsection{D products.}

The 2D products will consist of single images or collection of geometrically coherent images called 'series'. These images contain the red, green, blue and Nir bands at the same GSD and are proposed in three different resampling geometries and product levels.

The PRIMARY (L2A level) product corresponds to a perfect sensor geometry. The native geometric model is simplified to correct the matrix distortion and rolling-shutter effects, the attitude disturbances and spectral band co-registrations. A RPC model is provided in the metadata, added to the physical geometric model. The GSD for nadir viewing is $50 \mathrm{~cm}$. This GSD varies across the sampling grid and with the viewing conditions.

The PROJECTED (L2B level) product correspond to a projection at a constant elevation value over the WGS84 ellipsoid, generally the mean ground elevation value over the area. This elevation may be imposed by a user request. The image is resampled at a fixed $50 \mathrm{~cm}$ GSD whatever the actual viewing conditions.

The ORTHO (L2C level) product is similar to the PROJECTED product but with a projection on a SRTM-class DEM allowing the correction of acquisition and terrain off-nadir effects. The image is resampled in a geodetic or cartographic projection system at a $50 \mathrm{~cm}$ GSD whatever the actual viewing conditions. All the 2D products should contain a land-cover mask including a few classes such as water, vegetation and bare-soil. The completeness of these masks will depends on the state-of-the-art studies currently on-going.

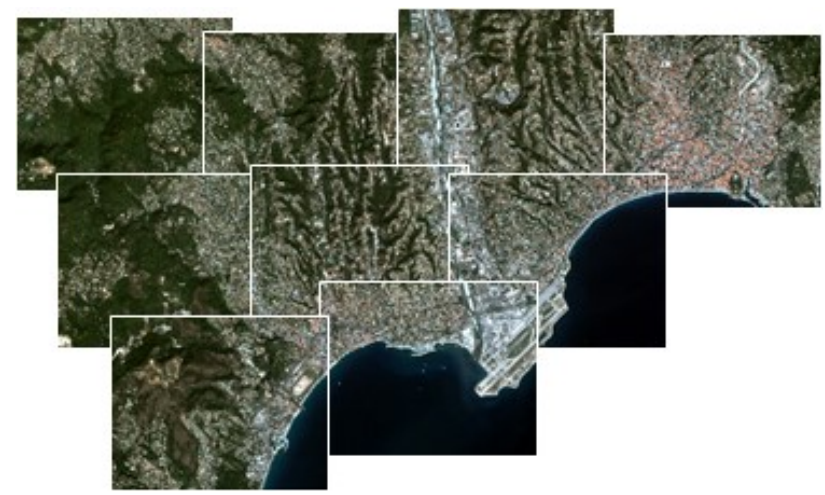

Figure 10.2D product series 
For the 2D product series, an additional bundle-adjustment step is processed to ensure the geometrical consistency. These series will be suited to time series, change detection or mosaics application. It could also be useful for end-users interested in $3 \mathrm{D}$ reconstruction with their own processing pipeline from multi-viewing acquisition conditions (stereo, tri-stereo, etc...).

\subsection{D products}

The $3 \mathrm{D}$ products of the core mission concern the LOCALDATED-DSM (L3 Level) and GLOBAL-DEM (L4 Level). Both levels are geometrically coherent multi-resolution raster DSM. The resolutions addressed should be $1 \mathrm{~m}, 5 \mathrm{~m}, 10 \mathrm{~m}$ and $15 \mathrm{~m}$ GSD. Works in progress aim at providing DTM (Digital Terrain Model) instead of DSM for GSD equal to $5 \mathrm{~m}$ and higher. The DSM will respect the DGED format in order to respect the governmental needs and most of high-resolution $3 \mathrm{D}$ applications interface.

The full-automatic process will include the filling of voids that could come from occluded areas (clouds, buildings or very sharp relief) or bad correlations (non-textured scenery or very noisy radiometry). It will also include the flattening of water bodies such as seas, lake or wide streams.

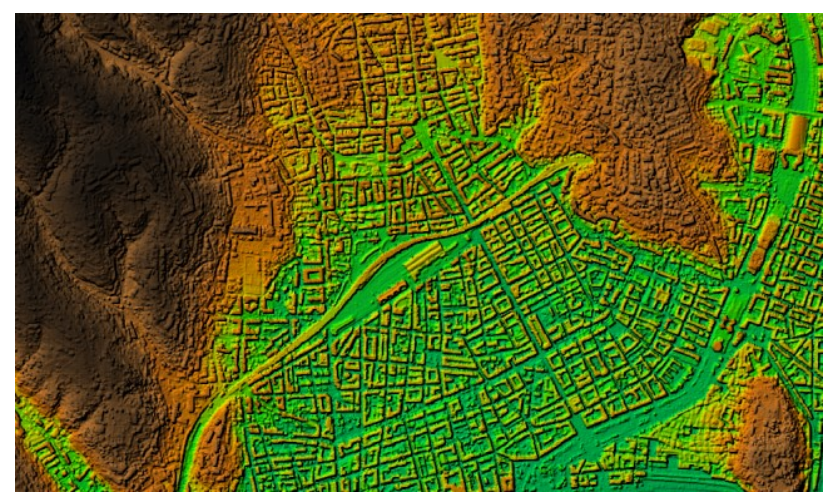

Figure 11. Example of CO3D Digital Surface Model (Nice downtown)

Many raster layers, perfectly superimposing each different resolutions DSM, will be provided within the products.

The first one will give the red, green, blue and Nir radiometry ortho-rectified on the concerned DSM.

The second one will give the genealogy of each pixel of the DSM, this means, whether it was or not a void before filling, and if so, the nature of the void (occlusion, bad correlation, ...) and the filling method applied (interpolation, external data, and so on).

Then, a performance mask will give the relative altimetric accuracy based mainly on the acquisition configuration $(\mathrm{B} / \mathrm{H}$ and viewing angles) and the local slope. A confidence mask will be associated to this performance mask taking into account, for instance, the number of 3D points included in the raster cell or some of the correlation process criteria. The information concerning the absolute altimetric performance will be provided in another mask sampled at a lower scale as this component vary with very low behaviour depending on the SRP distribution.

Another mask is a time stamp layer that will give the begin and end acquisition date of images concerned by the pixel 3D reconstruction.

Finally, the land-cover mask that should be available in the $2 \mathrm{D}$ products would be sampled in the DSM geometry. This last layer would be very useful for interpretation of the 3D data and also for the derivation of a DTM from the DSM in end-user post-processing.

On user demands, the 3D product will also include the highresolution (50 cm GSD) perfectly coherent $2 \mathrm{D}$ product series used to generate the $3 \mathrm{D}$ data. The level of these $2 \mathrm{D}$ products can be PRIMARY, PROJECTED or ORTHO. In the last case, the ortho-rectification will be processed thanks to the $10 \mathrm{~m}$ GSD endogenous DSM of the 3D product to ensure the best geometrical and temporal coherency between the $2 \mathrm{D}$ and $3 \mathrm{D}$ data. The $10 \mathrm{~m}$ version (currently under discussion) of the DSM seems to be the best choice to limit the ortho-rectification artifacts and to preserve high accuracy of the 3D data.

The LOCAL-DATED-DSM (L3) is dedicated to specific productions limited to regional areas, typically smaller than $2500 \mathrm{~km}^{2}$. The key feature of this product is the dating of the $3 \mathrm{D}$ data as the period of stereo acquisitions is one part of the user request. This dimension is of major interest for scientist working on earth surface kinematic behavior such as glacier melting or land sliding. More globally, in order to help the users with $3 \mathrm{D}$ change detection applications, it will be possible for the users to order L3 series corresponding to a collection of L3 products, each individually time stamped to a specific period, but all processed in the same pipeline to ensure the best geometric coherency thanks to a global bundle-adjustment. This opportunity will free the end-users with the hashing job of DSM post-registration. Some other specific parameters will also be part of the production order, such as the geographic or cartographic projection, the elevation reference (ellipsoid or geoid), and the elevation encoding format. It will also be possible to order an export of the $3 \mathrm{D}$ point-cloud computed before the DSM rasterization.

The GLOBAL-DEM (L4) is the worldwide 3D products coverage, split amongst a $0.25^{\circ} \times 0.25^{\circ}$ tiles mesh. Compared to the $\mathrm{L} 3$ products, the $\mathrm{L} 4$ processing parameters are imposed for most of them. The projection will be geographic and the elevation reference will be the geoid. The main challenge of the L4 tiles processing is to ensure a good geometrical coherency at the tiles border, this means the best planimetric junction and the limitation of elevation steps. The overall production strategy aims at reaching that goal as well as providing an accurate absolute location and altimetry of the 3D data at a large scale. This strategy takes into account, for each tile, the processing of massive bundle-adjustment centered on the tile, and covering margins on neighboring tiles. The size of these margins depends on the density of the available GCP of the SRP database in the region of interest. Figure 8, gives an example of tile production and bundle-adjustment margins in the case of dense GCP availability.

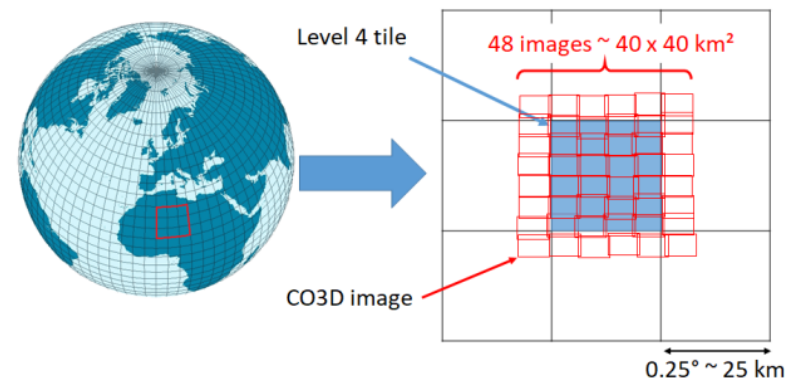

Figure 12. GLOBAL-DEM tiles production strategy (example) 


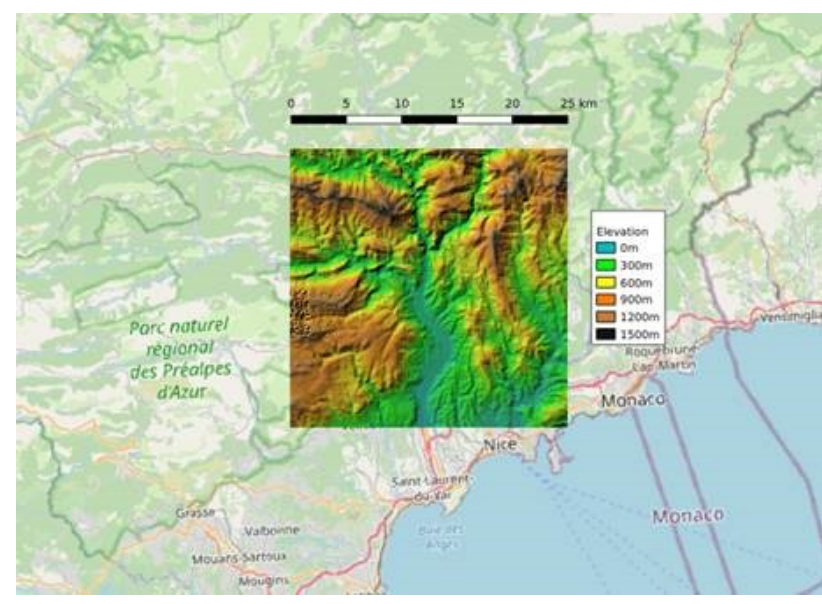

Figure 13. Example of one GLOBAL-DEM tile

\subsection{Products calibration and Quality Control}

The $2 \mathrm{D}$ product quality assessment will be conducted by CNES during the IOT Image Quality commissioning. In parallel of the calibration operations, several activities will concern the radiometric corrections and geometric model refinement, including the accurate cartography of the focal plane and the tuning of the attitude restoration processing. These activities are essential for the quality of the $3 \mathrm{D}$ products derived from the $2 \mathrm{D}$ products.

Then, the E2p demonstration phase, will be mainly dedicated to the $3 \mathrm{D}$ products quality control and processing parameters tuning. The activities will be conducted by the CNES with the support and the means of IGN.

Several methods are currently under study to assess the quality of the DSM. Some are suited to application on parsimonious sites distributed on various kind of sceneries. Some are more suited to exhaustive control of the automatic operational production. Some of the methods use benchmark, some other don't.

France coverage has been chosen for the demonstration phase because IGN has many high-resolution benchmarks coming from aerial or Lidar acquisitions. One of the tools used by CNES computes dense statistics over the difference between a DSM to qualify and a reference DSM. The comparison of DSM generated from different kinds of sources isn't an easy task and many cautions will have to be taken into account regarding the above-the-terrain structure for instance.

A second method, used in the PACA benchmark, consist in comparing the elevation of the DSM to elevation manually reconstructed from stereo image pairs on specific objects such as mountain crests, roads, shoreline and so on. This method is not exhaustive and expensive but conceivable for a few cases.

Another methods needs, for the site to expertise, additional acquisitions, one with an off-nadir view, let's say with a viewing angle higher than $20^{\circ}$, and a second one with nadir conditions. The nadir image gives a reference of a perfect orthoimage of the site. The off-nadir image is then ortho-rectified by the DSM to qualify and the resulting ortho-image is compared to the reference ortho-image on some specific objects or globally, thanks to dense correlation. Most of the differences between the two ortho-images come from the DSM imperfections.

Others methods will be studied with IGN in the following phases of the program to automatically identify potential defects on the DSM and alert the operators for the production monitoring.

All of these methods will also contribute to the building of the performance masks associated with the $3 \mathrm{D}$ products.

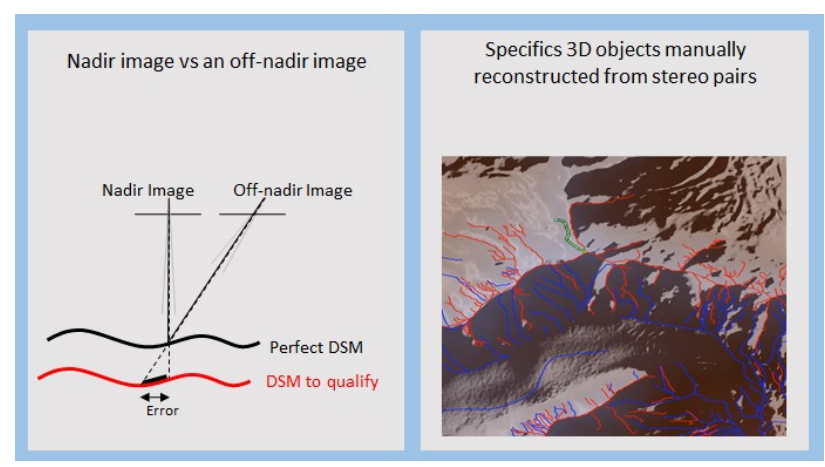

Figure 14. Examples of DSM Quality Control methods

\section{CONCLUSIONS}

Close to the end of the definition phase, CNES and ADS, with the support of IGN, converge to the specification of the CO3D system that should be operational in 2023 . This system, is very ambitious as it is based on a new generation of low-cost satellites and full-automatic ground processing to ensure its core mission, the creation of a worldwide DEM with high accuracy. This challenge becomes conceivable thanks to many innovations concerning the spatial and ground segments and also thanks to the permanent dialogue with the end-users to fulfil their needs in 3D products.

\section{REFERENCES}

Chandelier, L. Coeurdevey, L, 2020. A worldwide 3D GCP Database inherited from 20 years of massive multi-satellite observations, ISPRS 2020, Nice

Cournet, M., Sarrazin, E., 2020. Ground truth generation and disparity estimation for optical satellite imagery. ISPRS 2020, Nice

Hirschmuller, H., 2008, Stereo Processing by Semiglobal Matching and Mutual Information. IEEE Transactions on Pattern Analysis and Machine Intelligence, 30(2), 328-341.

Melet, O, Youssefi, D, 2020. CO3D mission digital surface model production pipeline, ISPRS 2020, Nice

Michel, J, Sarrazin, E, 2020. A new satellite imagery stereo pipeline designed for scalability, robustness and performance., ISPRS 2020, Nice

Youssefi, D. Michel, J, 2020. CARS: A photogrammetry pipeline using Dask graphs to construct a global 3D model', IGARSS 2020, Honolulu

Revised May 2020 